\title{
MODIFICAÇÕES VOCAIS ACÚSTICAS ESPECTROGRÁFICAS PRODUZIDAS PELA FONAÇÃO REVERSA
}

\author{
Spectrography acoustic vocal modifications \\ produced by reverse phonation
}

\author{
Valquíria Zimmer ${ }^{(1)}$, Carla Aparecida Cielo ${ }^{(2)}$, Leila Susana Finger ${ }^{(3)}$
}

\begin{abstract}
RESUMO
Objetivo: conhecer os efeitos da técnica de fonação reversa, realizando análise da sua eficácia sobre vozes femininas normais, por meio da espectrografia acústica de banda larga e de banda estreita. Métodos: 32 mulheres adultas jovens, que assinaram o Termo de Consentimento Livre e Esclarecido da pesquisa, submeteram-se à avaliação otorrinolaringológica e triagem fonoaudiológica a fim de eliminar possíveis alterações que pudessem interferir nos resultados da pesquisa. Tiveram amostras vocais coletadas antes e após realizar três séries de 15 repetições de fonação reversa em tempo máximo de fonação com tom e intensidade habituais, com 30 segundos de repouso passivo entre cada série. Utilizou-se o software Real Time Spectrogram (Kay Elemetrics Corp.) para gerar espectrogramas de banda larga e de banda estreita. Os dados obtidos foram analisados por meio de porcentagem simples e do teste do Qui-Quadrado, com nível de significância de 5\%. Resultados: houve tendência percentual ao aumento da definição de harmônicos, da regularidade do traçado, da intensidade e da definição dos Formantes 3 e 4, da intensidade das altas frequências e em todo 0 espectro; e aumento significante da definição do Formante 1. Conclusão: acredita-se que a técnica favoreça o alongamento e a vibração das pregas vocais com possível homogeneização e modificações ocorridas na mucosa, e conseqüente melhoria do sinal vocal, com maior regularidade de vibração e melhora de ressonância.
\end{abstract}

DESCRITORES: Voz; Espectrografia; Fonoterapia; Qualidade da Voz; Treinamento da Voz

\section{INTRODUÇÃO}

A voz exerce sobre o homem um papel de extrema importância uma vez que é considerada essencial para a comunicação e os relacionamentos ${ }^{1}$. Desse modo, a voz faz parte do cotidiano da sociedade, nos diferentes papéis desempenhados pelas pessoas no convívio social, como resultado de uma combinação de fatores biológicos, psicológicos e sociais ${ }^{2}$.

(1) Aluna do Curso de Graduação em Fonoaudiologia da Universidade Federal de Santa Maria, UFSM, Santa Maria, RS.

(2) Fonoaudióloga; Professora Adjunta de Graduação e PósGraduação do Departamento de Fonoaudiologia da Universidade Federal de Santa Maria, UFSM, Santa Maria, RS; Doutora em Linguística Aplicada pela Pontifícia Universidade Católica do Rio Grande do Sul.

(3) Fonoaudióloga da Prefeitura Municipal de Portão, RS; Mestre em Distúrbios da Comunicação Humana pela Universidade Federal de Santa Maria.

Conflito de interesses: inexistente
O fonoaudiólogo, por sua vez, é considerado o profissional que possui a maior capacitação para atuar na reabilitação vocal, no trabalho com o complexo processo fonatório o qual compreende o conhecimento anatômico e fisiológico. Considerando-se que o fonoaudiólogo é um profissional da voz e é habilitado a tratar a voz, cabe a ele atuar com os demais profissionais que têm como ferramenta diária a voz. Nesse caso, o embasamento teórico é fundamental para a atuação na prevenção, reabilitação e aperfeiçoamento vocal, uma vez que o conhecimento sobre as técnicas vocais é essencial ao clínico ${ }^{1}$.

A rotina fonoaudiológica abrange diversas técnicas fonoterapêuticas, que precisam ter sua fisiologia e efeitos compreendidos para o sucesso do tratamento. Portanto, a importância de comprovar a eficácia dessas técnicas é essencial para nortear o profissional sobre quais objetivos realmente poderá atingir durante sua prática ${ }^{3-6}$ 
A fonação reversa é uma das técnicas usadas no tratamento fonoterapêutico da $\mathrm{voz}^{4,7,8}$. Além de fazer parte do processo terapêutico, atua como importante recurso no diagnóstico diferencial de lesões de massa e auxilia a identificação de cistos nas pregas vocais ${ }^{7-10}$.

Durante a execução desta técnica vocal, a produção da voz combinada ao ato inspiratório propicia o alongamento da musculatura tireoaritenóidea (TA). Acredita-se que a atividade do músculo cricotireóideo (CT), durante a inspiração, favoreça o aumento da tensão de estiramento das pregas vocais na realização da fonação reversa. Consequentemente, ocorre a diminuição da contração da musculatura dos feixes do TA, o aumento da distância entre as pregas vestibulares e uma maior facilitação quanto à vibração da mucosa ${ }^{4}$.

As pesquisas e estudos abrangendo a eficácia das técnicas vocais são escassos, principalmente na literatura nacional. Então, há a necessidade de explorar minuciosamente essa área. A fonação reversa é uma das técnicas sobre a qual existem poucos estudos visando aos seus efeitos, quando usada na fonoterapia ${ }^{4,7,8}$.

A espectrografia acústica possibilita a análise da onda acústica e de seus componentes básicos. Desse modo, proporciona informações quanto à fonte glótica, a postura do trato vocal e as características das vogais e consoantes. O registro visual proveniente da análise da onda por meio da espectrografia sonora apresenta a distribuição da energia na freqüência e no tempo, possibilitando a visualização da gravação do ruído ${ }^{5,11-15}$. Ressaltase que os componentes espectrais pertencentes às alterações vocais podem estar relacionados às diferentes qualidades vocais e às características de distribuição de energia acústica ${ }^{16}$, sendo que o mesmo pode ocorrer na análise da eficácia das técnicas vocais.

Portanto, o objetivo deste trabalho é conhecer os efeitos da técnica de fonação reversa, realizando uma análise da sua eficácia sobre vozes femininas normais, por meio da espectrografia acústica de banda larga e de banda estreita.

\section{MÉTODOS}

Este estudo se caracterizou por ser uma investigação de campo exploratória, de cunho quantitativo e qualitativo. Participaram 32 mulheres adultas, brasileiras, sem queixas vocais e com ausência de patologias laríngeas. Os critérios de inclusão dos sujeitos para a participação na pesquisa foram: sexo feminino e idades entre 18 e 40 anos. Com esse último critério, o intuito foi descartar possíveis alterações hormonais e estruturais do aparelho fona- dor, já que processos, como o da mudança vocal e do envelhecimento, são comuns em faixa etária inferior ou superior à determinada nesta pesquisa.

Os critérios de exclusão foram: a) apresentar alguma alteração orgânica e/ou funcional ao nível laríngeo, pois distúrbios ao nível de prega vocal poderiam comprometer os resultados da pesquisa; b) apresentar queixa vocal como falha frequente na voz, rouquidão, ardência na garganta ou fadiga vocal, o que poderia sugerir indícios de alteração vocal orgânica e/ou funcional, podendo interferir na execução da técnica de fonação reversa; c) apresentar alterações decorrentes de distúrbios neurológicos, endocrinológicos ou psiquiátricos que pudessem dificultar a compreensão das ordens e a performance vocal durante as avaliações; d) apresentar alterações hormonais devido ao período prémenstrual, menstrual, menopausa ou gravidez; pois poderiam causar modificações nas pregas vocais e influenciar na execução da técnica de fonação reversa; e) apresentar alergias respiratórias como sinusite e rinite ou estar com gripe no dia das avaliações, fatores esses que poderiam limitar 0 desempenho vocal do sujeito por prováveis modificações nas pregas vocais; f) apresentar hábito de tabagismo e/ou etilismo; pois tais agentes causam irritação do trato vocal e podem levar à formação de patologias laríngeas; g) apresentar queixas ou alterações auditivas, pois podem dificultar o automonitoramento da voz; h) apresentar alterações do sistema estomatognático ou das funções neurovegetativas; i) apresentar dificuldades na realização da técnica de fonação reversa; j) cantar ou já ter participado de coros, pois os sujeitos teriam conhecimentos sobre técnicas vocais e treinamento de voz; k) ter realizado fonoterapia e/ou tratamento otorrinolaringológico prévio, a fim de evitar condicionamento vocal e a presença ou histórico de alguma patologia laríngea; I) apresentar conhecimento sobre a técnica de fonação reversa.

Para garantir os aspectos bioéticos do estudo, todas as participantes assinaram o termo de consentimento livre e esclarecido (TCLE), como recomenda a norma 196/96 da Comissão Nacional de Ética em Pesquisa - CONEP/1996. Em seguida, foram realizadas as avaliações, a fim de averiguar se todos os participantes se adequavam aos critérios de inclusão e de exclusão estabelecidos.

Primeiramente, foi solicitado às participantes que respondessem a um questionário elaborado pelas pesquisadoras com 0 intuito de investigar queixa de alteração vocal e outros aspectos que pudessem interferir na qualidade vocal, conforme os critérios de exclusão descritos. Após o preenchimento, as participantes foram encaminhadas à avaliação otorrinolaringológica, por meio do exame 
laringoscópico indireto, objetivando-se descartar a presença de patologias e ou disfunções laríngeas. Realizou-se avaliação do sistema e das funções estomatognáticas, a fim de verificar a presença de alterações que pudessem comprometer a articulação da fala e a qualidade da voz. Por fim, foi realizada a avaliação auditiva, sendo testados os tons puros pela via aérea nas frequências de 250 , $500,1000,2000,4000,6000$ e $8000 \mathrm{~Hz}$ a $25 \mathrm{~dB}$, em cabine acusticamente tratada, com audiômetro modelo Fonix FA 12 Digital

Apresentaram-se como voluntárias 48 mulheres, das quais 16 foram excluídas da pesquisa e, posteriormente, encaminhadas para avaliações detalhadas. Foram excluídas: cinco mulheres na aplicação do questionário (duas por serem fumantes e três por terem distúrbios alérgicos); oito por não terem disponibilidade em realizar a avaliação ORL; uma por ter mais de 40 anos; e duas por serem menores de 18 anos.

As 32 voluntárias selecionadas, com idades entre 18 e 39 anos (média de 20,56 anos), iniciaram individualmente a coleta de dados com a emissão sustentada da vogal /a/. Nas gravações, foi utilizado microfone acoplado ao gravador digital da marca Creative Labs modelo MuVo Tx FM, as participantes permaneceram em pé, com os braços estendidos ao longo do corpo, o microfone posicionado próximo à boca, a uma distância de $4 \mathrm{~cm}$ em um ângulo de $90^{\circ}$ graus ${ }^{11,14,15}$. Após o posicionamento correto, foi solicitado que inspirassem profundamente e mantivessem a emissão sustentada sem o uso de ar de reserva expiratória, em frequência e intensidade habituais, emitindo o som em tempo máximo de fonação (TMF).

Em seguida, receberam orientações para realizar três séries de fonação reversa com 15 repetições cada ${ }^{3-5,17}$, com inspirações nasais. Para a realização da técnica as participantes foram orientadas a esvaziar os pulmões e realizar o "ihn" durante a inspiração nasal, sendo monitoradas, pois, sempre que necessário, foram realizadas correções para a emissão correta da técnica, sem diferenças entre os sujeitos.

As participantes executaram a técnica na posição sentada, com a coluna ereta, para não haver deslocamento cervical, e mantendo os pés apoiados no chão; mantiveram também ritmo entre a execução dos exercícios e realizaram repouso passivo, ou seja, silêncio absoluto por 30 segundos após cada série de 15 repetições ${ }^{3-5,17}$. A ingestão de água durante a coleta foi liberada. Ao finalizar as três séries da técnica, foi novamente coletada a emissão sustentada da vogal/a/, nas mesmas condições iniciais.
A análise acústica constou da extração dos 3,5 segundos iniciais da gravação da emissão sustentada da vogal/a/, excluindo-se o ataque vocal para que não interferisse na análise dos dados ${ }^{12}$. Posteriormente, as vozes foram analisadas por meio do programa Real Time Spectrogram da Kay Elemetrics Corp, o qual forneceu os traçados espectrográficos de banda larga filtrados em 100 points (646 $\mathrm{Hz}$ ), e de banda estreita com o filtro de 1024 points $(63 \mathrm{~Hz}$ ); sendo a taxa de amostragem de $11 \mathrm{KHz}$ e a quantificação de 8 bits.

A análise dos traçados espectrográficos contou com a avaliação de três fonoaudiólogas com experiência na área de voz, considerando-se, para fins de resultado, a opinião predominante entre as juízas. Salienta-se que as juízas realizaram as avaliações individualmente sem entrar em consenso uma com a outra. Quando necessário, uma quarta juíza atuou como critério de desempate.

Cada conjunto de espectrografias, de banda larga e de banda estreita da mesma voluntária, foi duplicado, codificado em "antes" e "após" a execução da técnica, e entregue às juízas para comparação, sem que as mesmas soubessem que avaliariam duas vezes os dados da mesma participante, com o intuito de se obter maior consistência e confiabilidade quanto aos resultados da avaliação. Foi utilizado apenas um protocolo de avaliação espectrográfica, no qual as juízas assinalaram as modificações observadas na comparação da espectrografia anterior à realização da técnica com a espectrografia pós-técnica.

Foram avaliados os seguintes aspectos em filtro de banda larga (EBL): intensidade dos formantes (F1, F2, F3 e F4); intensidade das altas frequências; intensidade em todo o espectro vocal; presença de ruído em todo espectro vocal, bem como nas frequências altas, médias e baixas; largura de banda dos formantes (F1, F2, F3 e F4); definição dos formantes (F1, F2, F3 e F4); e a presença de anti-ressonância/damping em todo o espectro vocal. Todos esses aspectos foram classificados em redução, aumento ou sem alteração. Analisou-se, também, a regularidade do traçado, classificada em maior, menor ou sem alteração.

$\mathrm{Na}$ análise em filtro de banda estreita (EBE), foram considerados os aspectos: intensidade das altas frequências; intensidade em todo o espectro vocal; presença de ruído em todo espectro vocal, bem como nas frequências altas, médias e baixas; e a presença de anti-ressonância/damping em todo o espectro vocal; sendo tais aspectos também classificados em redução, aumento, ou sem alteração. Analisou-se, também, a definição de harmônicos e a regularidade do traçado, classificados em maior, 
menor, ou sem alteração assim como na análise anterior.

Esta pesquisa foi previamente aprovada pelo Comitê de Ética em Pesquisa da instituição de origem (no 23081.008439/2007-16; 0087.0.243.000-07).

Os dados obtidos por meio das avaliações foram tabulados e analisados estatisticamente por meio de porcentagem simples e do teste Qui-Quadrado, com nível de significância de $5 \%(p<0,05)$.

\section{RESULTADOS}

Os resultados da EBL, após a utilização da fonação reversa, são apresentados na Tabela 1 ao nível de significância de $5 \%$.

Os resultados da EBE, após a utilização da técnica de fonação reversa, são apresentados na Tabela 2, ao nível de significância de 5\%.

Tabela 1 - Resultado da análise acústica do filtro de banda larga (EBL) após a fonação reversa

\begin{tabular}{|c|c|c|c|c|c|c|c|c|c|}
\hline \multirow[t]{2}{*}{ EBL } & \multicolumn{2}{|c|}{ Redução } & \multicolumn{2}{|c|}{ Aumento } & \multicolumn{2}{|c|}{$\begin{array}{c}\text { Sem } \\
\text { Alteração }\end{array}$} & \multicolumn{2}{|c|}{ Ausente } & \multirow[t]{2}{*}{$\mathbf{p}$} \\
\hline & $\mathbf{n}$ & $\%$ & $\mathbf{n}$ & $\%$ & $\mathbf{n}$ & $\%$ & $\mathbf{n}$ & $\%$ & \\
\hline Intensidade do F1 & 3 & 9,38 & 12 & 37,5 & 17 & 53,13 & & & $0,0089^{*}$ \\
\hline Intensidade do F2 & 11 & 34,38 & 6 & 18,75 & 15 & 46,8 & & & 0,1486 \\
\hline Intensidade do F3 & 7 & 21,8 & 14 & 43,75 & 11 & 34,38 & & & 0,3147 \\
\hline Intensidade do F4 & 7 & 21,88 & 15 & 46,88 & 10 & 31,25 & & & 0,2163 \\
\hline Intensidade das altas freqüências & 6 & 18,75 & 15 & 46,88 & 11 & 34,88 & & & 0,1486 \\
\hline Intensidade em todo o espectro & 8 & 25 & 14 & 43,75 & 10 & 31,25 & & & 0,4169 \\
\hline Ruído em todo o espectro & 11 & 34,38 & 12 & 37,5 & 9 & 28,13 & & & 0,8035 \\
\hline Ruído nas altas frequências & 8 & 25 & 12 & 37,5 & 12 & 37,5 & & & 0,6065 \\
\hline Ruído nas médias frequências & 12 & 37,5 & 9 & 28,13 & 11 & 34,38 & & & 0,8035 \\
\hline Ruído nas baixas frequências & 4 & 12,5 & 5 & 15,63 & 23 & 71,88 & & & $0,0001^{*}$ \\
\hline Largura de banda do F1 & 2 & 6,25 & 12 & 37,5 & 18 & 56,25 & & & $0,0022^{*}$ \\
\hline Largura de banda do F2 & 6 & 18,75 & 6 & 18,75 & 20 & 62,5 & & & $0,0022^{*}$ \\
\hline Largura de banda do F3 & 6 & 18,75 & 7 & 21,88 & 19 & 59,38 & & & $0,0074^{*}$ \\
\hline Largura de banda do F4 & 3 & 9,38 & 12 & 37,5 & 17 & 53,13 & & & $0,0089^{*}$ \\
\hline Definição do F1 & 5 & 15,63 & 19 & 59,38 & 8 & 25 & & & $0,0061^{*}$ \\
\hline Definição do F2 & 11 & 34,38 & 10 & 31,25 & 11 & 34,38 & & & 0,9692 \\
\hline Definição do F3 & 8 & 25 & 15 & 46,88 & 9 & 28,13 & & & 0,2609 \\
\hline Definição do F4 & 5 & 15,63 & 16 & 50 & 11 & 34,38 & & & 0,0582 \\
\hline Regularidade do traçado & 7 & 21,88 & 15 & 46,88 & 10 & 31,25 & & & 0,3796 \\
\hline Anti-ressonância & 9 & 28,13 & 5 & 15,63 & 9 & 28,13 & 9 & 28,13 & 0,2357 \\
\hline
\end{tabular}

*significância estatística pelo Teste Qui-Quadrado

Tabela 2 - Resultado da análise acústica do filtro de banda estreita (EBE) após a fonação reversa

\begin{tabular}{|c|c|c|c|c|c|c|c|c|c|}
\hline \multirow[t]{2}{*}{ EBE } & \multicolumn{2}{|c|}{ Redução } & \multicolumn{2}{|c|}{ Aumento } & \multicolumn{2}{|c|}{$\begin{array}{c}\text { Sem } \\
\text { Alteração }\end{array}$} & \multicolumn{2}{|c|}{ Ausente } & \multirow[t]{2}{*}{$\mathbf{p}$} \\
\hline & $\mathbf{n}$ & $\%$ & $\mathbf{n}$ & $\%$ & $\mathbf{n}$ & $\%$ & $\mathbf{n}$ & $\%$ & \\
\hline Intensidade das Altas Frequências & 6 & 18,75 & 13 & 40,63 & 13 & 40,63 & & & 0,2163 \\
\hline Intensidade em todo o espectro & 7 & 21,88 & 12 & 37,5 & 13 & 40,63 & & & 0,3796 \\
\hline Ruído em todo o espectro & 8 & 25 & 12 & 37,5 & 12 & 37,5 & & & 0,6065 \\
\hline Ruído nas altas frequências & 4 & 12,5 & 11 & 34,38 & 17 & 53,13 & & & $0,0189^{*}$ \\
\hline Ruído nas médias frequências & 6 & 18,75 & 10 & 31,25 & 16 & 50 & & & 0,0930 \\
\hline Ruído nas baixas frequências & 4 & 12,5 & 6 & 18,75 & 22 & 68,75 & & & $0,0001^{*}$ \\
\hline Definição de Harmônicos & 8 & 25 & 15 & 46,88 & 9 & 28,13 & & & 0,2609 \\
\hline Regularidade do traçado & 7 & 21,88 & 12 & 37,5 & 13 & 40,63 & & & 0,3796 \\
\hline Anti-ressonância & 6 & 18,35 & 7 & 21,88 & 13 & 40,63 & 6 & 18,75 & 0,2357 \\
\hline
\end{tabular}

*significância estatística pelo Teste Qui-Quadrado 


\section{DISCUSSÃO}

Neste estudo, evidenciou-se que, após realização da técnica de fonação reversa, os resultados foram estatisticamente significantes quanto a não alteração da largura de banda de F1, F2, F3 e F4 (Tabela1). Esse resultado justifica-se devido não haver modificação da postura do trato vocal durante a coleta da emissão habitual da vogal /a/, tendo sido realizada a mesma vogal pré e pós-execução da técnica de fonação reversa.

A ressonância é proveniente das estruturas supraglóticas do trato vocal, sendo dependente da amplitude, da frequência de oscilações e do comprimento da onda sonora. Os formantes são, então, resultantes da transformação do sinal sonoro produzido pela fonte glótica quando recebe influência do trato vocal ou filtro ${ }^{18}$.

Quanto à divisão dos formantes, as frequências de $\mathrm{F} 1$ se relacionam com a variação da posição da língua associada à abertura da mandíbula, no plano vertical. As frequências de F2 mostram o grau de variação da língua no sentido ântero-posterior, evidenciando sua mobilidade no plano horizontal. O F3 se relaciona às cavidades à frente e atrás do posicionamento da língua. As frequências de F4 estão relacionadas ao formato e à altura da laringe e da faringe ${ }^{11,18-20}$.

A identidade fonética da vogal procede das frequências dos três primeiros formantes, sendo que os dois primeiros formantes contribuem de maneira mais acentuada ${ }^{18,19}$

Em pesquisa realizada com a técnica do som hiperagudo também não se evidenciaram modificações quanto à largura de banda dos formantes ${ }^{5}$. Além disso, a manutenção da largura de banda dos formantes, obtida no presente estudo, relaciona-se com a manutenção dos níveis de ruído.

A postura da língua durante a realização da vogal /a/ em registro modal, utilizada no presente estudo, encontra-se rebaixada no plano vertical e centralizada no plano horizontal. No entanto, os ajustes supralaríngeos necessários à realização da fonação reversa, ou seja, a emissão do "ihn", no momento da inspiração, favorece a produção de frequências agudas, sendo a vogal /i/ classificada como alta, por apresentar a língua em posição elevada no plano vertical, com menor abertura mandibular.

Nesta pesquisa, nota-se que a intensidade do F1 na vogal /a/, após a execução da técnica de fonação reversa, não apresentou alterações significantes. No entanto, pode-se verificar que houve aumento percentual da intensidade e da definição de F3 e F4, paralelamente ao aumento significante da definição de F1 (Tabela 1). Esses resultados estão de acordo com aqueles da pesquisa realizada com a técnica do som hiperagudo, na qual também se evidenciou aumento da intensidade de F3 e F4 ${ }^{5}$. A realização da técnica do som hiperagudo também exige a emissão da vogal /i/, a mesma vogal utilizada no presente estudo na produção do "ihn", portanto, a postura do trato vocal em ambas as técnicas é similar, podendo estender seu efeito à produção posterior do /a/ em registro modal.

$\mathrm{O}$ resultado quanto à definição do $\mathrm{F} 1$ é similar a outro estudo ${ }^{21}$ que avaliou as modificações ocorridas após a terapia fonoaudiológica de indivíduos com diagnóstico de disfonia funcional e verificou melhora acústica, embora sem significância do F1, evidenciando o papel positivo das técnicas vocais.

A fonação reversa promove a mobilização das estruturas supraglóticas, favorecendo a diminuição da constrição mediana e ântero-posterior ${ }^{4}$.

Os resultados do presente estudo que verificou tendência percentual de aumento da definição de harmônicos, da regularidade do traçado, da intensidade e da definição de F3 e F4, da intensidade das altas freqüências e em todo o espectro; e aumento significante da definição de F1, além da manutenção do ruído (Tabela 1 e 2) podem ser relacionados à interação fonte e filtro, pois o treinamento vocal com ênfase nas regiões agudas propicia estreitamento da supraglote e diminuição do fluxo transglótico de ar entre as pregas vocais que sofrem modificações em sua vibração. Há, portanto, diminuição da fase de fechamento das pregas vocais que proporciona aumento na intensidade dos harmônicos superiores da região de $3 \mathrm{KHz}{ }^{22}$. Isso converge com os resultados obtidos, pois a coleta da emissão de /a/ modal, no presente estudo, foi realizada imediatamente após a execução intensiva da técnica de fonação reversa (três séries de 15 repetições).

Contrariamente ao princípio da fonação expiratória, cuja pressão ocorre contra a gravidade, a técnica de fonação reversa corresponde à emissão de um som durante o ato inspiratório, no qual há a vibração ampla e sincrônica da mucosa a favor da gravidade. Na execução da técnica, a face superior das pregas vocais se relaciona com a queda da pressão transglótica. Dessa forma, é provável que a pressão traqueal negativa gere uma mudança de força vetorial, promovendo o deslocamento dos tecidos a favor da gravidade 4,10 .

Quando a mucosa vibra a favor da gravidade, favorece a renovação da camada de muco que reveste as pregas vocais, facilitando o deslizamento entre as pregas, e mobiliza a lâmina própria da mucosa, propiciando a mobilidade e a soltura da mesma. Dessa maneira, a partir da mucosa massageada, a circulação sanguínea periférica é ativada 
em toda extensão das pregas vocais, bem como sua homogeneização ${ }^{4}$.

Os resultados também são apoiados por pesquisa ${ }^{23}$ que identificou o formante do cantor entre $2,8-3,4 \mathrm{KHz}$, no agrupamento entre F3, F4 e F5 e que estaria relacionado com a percepção de "briIho" e de projeção vocal, e são ainda corroborados por outro estudo sobre a qualidade vocal de estudantes universitários do sexo masculino durante a fala ${ }^{24}$ que verificou que vozes consideradas boas se caracterizam por um espectro menos íngreme e picos proeminentes nas frequências de 3 e $4 \mathrm{KHz}$.

Neste estudo, com relação aos resultados da EBL (Tabela 1), pode-se observar aumento percentual da intensidade em todo o espectro e nas altas frequências, após a execução da técnica de fonação reversa. Essa técnica é resultante da elevada pressão supraglótica que auxilia o efeito de Bernoulli, devido à entrada de ar e à mobilização da mucosa ${ }^{4,10}$, promovendo o adequado fechamento glótico que se relaciona intimamente com a amplitude/intensidade da onda sonora ${ }^{11,24-28}$. A intensidade em todo o espectro e nas altas frequências também está relacionada ao tônus da laringe e à resistência glótica ${ }^{29,30}$.

Esses resultados vão de encontro aos do estudo sobre a análise comparativa das medidas espectrográficas da voz antes e depois da fonoterapia ${ }^{30}$ que constatou a não modificação do grau de escurecimento do traçado após a fonoterapia, na maior parte das vozes analisadas. No entanto, o fato dos sujeitos provavelmente não apresentarem alteração da loudness, antes do processo terapêutico, pode justificar esses resultados.

A presente pesquisa verificou que não houve alteração quanto à presença de ruído nas baixas frequências e que houve a manutenção percentual de ruído nas demais frequências e em todo o espectro (Tabela 1 e 2). Isso, juntamente com as melhoras observadas em F3 e F4, na intensidade em todo o espectro, na intensidade das altas frequências, na regularidade do traçado, na definição de harmônicos, sugere o benefício da fonação reversa sobre a fonte glótica, uma vez que, segundo pesquisas prévias ${ }^{11,16}$, a presença de ruído é relacionada à presença de rouquidão e à aperiodicidade de vibração das pregas vocais.

Em um estudo ${ }^{30}$, houve melhora significante no que tange à presença de ruído, com a diminuição do mesmo após a fonoterapia talvez devido ao fato de a reavaliação ter ocorrido após um tempo maior de fala habitual e por se tratar de indivíduos disfônicos que já apresentavam vozes alteradas. A literatura mostra que sujeitos que apresentam vozes disfônicas evidenciam melhoras mais significantes quanto ao ruído, do que os indivíduos que mostram vozes mais próximas do normal, como no presente estudo no qual os níveis de ruído praticamente se mantiveram ${ }^{31}$.

Quanto à definição de harmônicos, houve aumento percentual na EBE (Tabela 2) e a regularidade do traçado apresentou aumento percentual somente na EBL (Tabela 1). Mesmo não apresentando significância estatística, tais resultados sugerem que a realização da fonação reversa favorece a mobilização da mucosa das pregas vocais e essa massagem na sua cobertura promove a homogeneização interna e a renovação do muco existente na camada epitelial que a reveste. Dessa forma, há melhora na ressonância, proveniente de um sinal glótico mais harmônico, menos ruidoso, de propagação eficiente e modificações adequadas no trato vocal ${ }^{16}$ Portanto, a técnica de fonação reversa favorece a presença de maior número de harmônicos, com maior definição durante a emissão fonatória ${ }^{4}$.

$\mathrm{Na}$ pesquisa com o som hiperagudo, verificaram-se resultados semelhantes quanto à definição de harmônicos e à regularidade do traçado com aumento estatisticamente significante quanto à regularidade do traçado na $E B L$ e na EBE ${ }^{5}$. O mesmo ocorrendo com outro estudo ${ }^{30}$ que evidenciou melhora significante na estabilidade do traçado espectrográfico e na presença de harmônicos e constatou não haver diferença estatística na condição pós-fonoterapia para a forma do traçado na $E B E$, devido à ausência de irregularidade no traçado espectrográfico antes do início da terapia.

Em pesquisa ${ }^{3}$ sobre as modificações laríngeas e vocais após a produção do som vibrante lingual, observaram-se resultados significativos quanto à EBL na maioria dos aspectos analisados. Verificouse aumento da intensidade dos formantes, aumento da intensidade em todo o espectro, maior definição dos formantes e maior regularidade do traçado, convergindo com os resultados do presente trabalho.

Em estudo realizado a partir das características espectrográficas de vozes roucas, ásperas e normais ${ }^{13}$, verificou-se que o espectrograma correspondente às vozes normais foi preenchido de harmônicos em sua quase totalidade. Portanto, a grande quantidade de harmônicos encontrada proporcionou maior nitidez à voz. Na presente pesquisa, o aumento percentual da definição de harmônicos, da intensidade de F3 e F4, e o aumento significativo de $\mathrm{F} 1$ reforçam o papel positivo da fonação reversa sobre o sinal vocal.

Os resultados encontrados neste estudo evidenciam que a produção da técnica de fonação reversa se mostrou eficiente na melhoria do sinal vocal, uma vez que beneficia o controle do ar inspirado e o efeito de Bernoulli. 
As modificações sobre a camada mucosa e de muco das pregas vocais as quais acarretam o fechamento glótico, a regularidade vibratória assim como a coordenação pneumofonoarticulatória favorecem, consequentemente, a melhora do sinal glótico e da ressonância pelo aumento de energia e de harmônicos no espectro, o que converge com os resultados do presente estudo 24,27-30.

Dentre as análises acústicas existentes, a espectrografia é um recurso complementar às avaliações fonoaudiológicas e ainda possibilita a análise visual e a comparação das evoluções do paciente durante e após o processo fonoterapêutico ${ }^{32}$. Em estudo com vozes disfônicas, utilizou-se a escala GRBAS e evidenciou-se que o auxílio visual oferecido pela análise espectrográfica aumenta a confiabilidade da avaliação, complementando a avaliação perceptivo-auditiva ${ }^{33}$, além de propiciar a avaliação de toda a extensão da produção vocal ${ }^{34}$.

\section{CONCLUSÕES}

As modificações vocais acústicas espectrográficas produzidas pela fonação reversa em vozes femininas normais mostraram que houve tendência percentual ao aumento da definição de harmônicos, da regularidade do traçado, da intensidade e da definição de F3 e F4, da intensidade das altas frequências e de todo o espectro. Também houve aumento significante da definição de F1.

Em função das mudanças espectrográficas observadas, acredita-se que esta técnica favoreça o alongamento e a vibração das pregas vocais com possível homogeneização e modificações ocorridas na mucosa. Consequentemente, há melhoria do sinal vocal, com maior regularidade de vibração e melhora de ressonância.

\begin{abstract}
Purpose: to improve knowledge on the effects of reverse phonation technique based on an analysis of its efficiency on normal female voice, using wideband and narrowband spectrograph. Methods: 32 young adult women who signed the Informed Consent were submitted to otorhinolaryngologic evaluation and speech and hearing screening in order to avoid alterations that could interfere in the results of the study; voice samples were collected before and after three series containing 15 reverse phonation repetitions, at maximum phonation time with habitual tone and intensity, and 30-seconds interval of passive rest between the series. We used the Real Time Spectrogram software (Kay Elemetrics Corp.) to generate wideband and narrowband spectrograph. Data were analyzed by means of the simple percentage and the Qui-Square test, with a significance level of $5 \%$. Results: there was a trend to increase in the definition of harmonics; an increase in the regularity the spectrograph; in the intensity and definition of Formants 3 and 4 frequencies; in the intensity of high frequencies and in the entire spectrum; as well as a significant increase in the definition of Formant 1. Conclusion: finally, we believe that the technique enables the stretching and vibration of the vocal folds, with a possible homogenization and modifications in the mucous, consequently improving the vocal signal with a higher vibration regularity and an improvement in resonance.
\end{abstract}

KEYWORDS: Voice; Spectrography; Speech Therapy; Voice Quality; Voice Training

\section{REFERÊNCIAS}

1. Pedroso MIL. Técnicas vocais para profissionais da voz [monografia]. São Paulo (SP): CEFAC Pós-Graduação em Saúde e Educação; 1997.

2. Roman G, Cielo CA. Particularidades da técnica fonoterapêutica de sons hiperagudos: revisão de literatura. Rev. CEFAC. 2006; 8(3):360-7.

3. Schwarz K. Modificações laríngeas e vocais produzidas pela técnica de vibração sonorizada de língua [dissertação]. Santa Maria (RS): Universidade Federal de Santa Maria; 2006.

4. Finger LS. Modificações vocais produzidas pela fonação reversa em laringes Normais [dissertação]. Santa Maria (RS): Universidade Federal de Santa Maria; 2008.

5. Roman-Niehues G. Modificações vocais decorrentes da técnica fonoterapêutica de sons hiperagudos [dissertação]. Santa Maria (RS): Universidade Federal de Santa Maria; 2008.

6. Bolzan GP, Cielo CA, Brum DM. Efeitos do som basal em fendas glóticas. Rev. CEFAC. 2008; 10(2):21825. dx.doi.org/10.1590/S1516-18462008000200011

7. Pastana SG, Gomes E, Castro L. Conduta fonoaudiológica e avaliação estroboscópica no diagnóstico diferencial do cisto. Rev. CEFAC. 
2007; 9(3):397-403. dx.doi.org/10.1590/S151618462007000300013

8. Finger LS, Cielo CA. Aspectos fisiológicos e clínicos da técnica fonoterapêutica de fonação reversa. Rev Bras Otorrinolaringol. 2007; 73(2): 271-7.

9. Kothe C, Schade G, Fleischer S, Hess M. Forced inspiration: a laryngoscopy-based maneuver to assess the size of Reinke's edema. Laryngoscope. 2003; 113(4):741-2.

10. Sulica L, Behrman A, Roark R. The inspiratory maneuver: a simple method to assess the superficial lamina propria during endoscopy. J Voice. 2005; 19(3):481-4.

11. Behlau M. Voz: o livro do especialista. Vol. I. Rio de Janeiro: Revinter; 2001. 348p.

12. Barros APB, Carrara-De Angelis E. Análise acústica da voz. In: Dedivitis RA, Barros APB. Métodos de avaliação e diagnóstico de laringe e voz. São Paulo: Lovise; 2002. p. 201-21.

13. Pontes PAL, Vieira VP, Gonçalves MIR, Pontes AAL. Características das vozes roucas, ásperas e normais: análise acústica espectrográfica comparativa. Rev Bras Otorrinolaringol. 2002; 68(2):182-8.

14. Deliyski DD, Shaw HS, Evans MK. Adverse effects of environmental noise on acoustic voice quality measurements. J Voice. 2005; 19(1):15-28.

15. Vieira MN, Rosa LLC. Avaliação acústica na prática fonoaudiológica. In: Pinho SMR, Tsuji DH, Bohadana SC. Fundamentos em laringologia e voz. Rio de Janeiro: Revinter; 2006. p. 33-52.

16. De Krom G. Some spectral correlates of pathological breathy and rough voice quality for different types of vowel fragments. J Speech Hear Res. 1995; 38(4):794-811.

17. Saxon KG, Scheneider CM. Vocal exercise physiology. California: Singular Publishing Group; 1995. 71p.

18. Magri A, Cukier-Blaj S, Karman DF, Camargo ZA. Correlatos perceptivos e acústicos dos ajustes supraglóticos na disfonia. Rev. CEFAC. 2007; 9(4):512-8. dx.doi.org/10.1590/ S1516-18462007000400012

19. Bele IV. The speaker's formant. J Voice. 2006; 20(4):555-78.

20. Rehder MIBC, Behlau M. Análise vocal perceptivo-auditiva e acústica, falada e

\section{DOI: 10.1590/S1516-18462010005000005}

RECEBIDO EM: 22/12/2008

ACEITO EM: 13/08/2009

Endereço para correspondência:

Valquíria Zimmer

Rua Coronel Antero Correa de Barros, 655/104

Santa Maria - RS

CEP: 97010-120

E-mail: valquiriazimmer@yahoo.com.br cantada de regentes de coral. Pró-Fono. 2008; 20(3):195-200.

21. Kitzing $P$, Akerlund $L$. Long-time average spectrograms of dysphonic voices before and after therapy. Folia Phoniatr. 1993; 45(2):53-61.

22. Master S, Biase N, Pedrosa V, Chiari BM. O espectro médio de longo termo na pesquisa e na clínica fonoaudiológica. Pró-Fono. 2006; 18(1):111-20.

23. Sunderg J. The science of the singing voice. Illinois: Northern Illinois University Press; 1987.

24. Leino $T$. Long-term average spectrum in screening of voice quality in speech: untrained male university students. J Voice. 2008; In Press, Corrected Proof.

25. Holmberg EB, Doyle P, Perkell JS, Hammarberg $B$, Hillman RE. Aerodynamic and acoustic voice measurements of patients with vocal nodules: variation in baseline and changes across voice therapy. J Voice. 2003; 17(3):269-82.

26. Henrich N, D'Alessandro C, Doval B, Castellengo M. Glottal open quotient in singing: measurements and correlation with laryngeal mechanisms, vocal intensity, and fundamental frequency. J Acoust Soc Am. 2005; 117(3):1417-30.

27. Master S, Biase N, Chiari BM, Laukkanen AM. Acoustic and perceptual analyses of Brazilian male actors' and nonactors' voices: long-term average spectrum and the "actor's formant". J Voice. 2008; 22(2):146-54.

28. Sergeant DC, Welch GF. Gender differences in long-term average spectra of children's singing voices. J Voice. 2009; 23(3):319-36.

29. Drumond LB, Gama ACC. Correlação entre dados espectrográficos e perceptivo-auditivos de vozes disfônicas. Fono Atual. 2006; 8(35):49-58.

30. Côrtes MG. Análise comparativa dos parâmetros espectrográficos da Voz antes e depois da fonoterapia [monografia]. Belo Horizonte (MG): Universidade Federal de Minas Gerais; 2007.

31. Speyer R, Wieneke GH, Dejonckere PH. Documentation of progress in voice therapy: perceptual, acoustic, and laryngostroboscopic findings pretherapy and posttherapy. J Voice. 2004; 18(3):325-40.

32. Nemr K, Amar A, Abrahão M, Leite GCA, Köhle $J$, Santos $A O$, et al. Análise comparativa entre avaliação fonoaudiológica perceptivo-auditiva, análise acústica e laringoscopias indiretas para avaliação vocal em população com queixa vocal. Rev Bras Otorrinolaringol. 2005; 71(1):13-7.

33. Martens JWMAF, Versnel $\mathrm{H}$, Dejonckere, $\mathrm{PH}$. The effect of visible speech in the perceptual rating of pathological voices. Arch Otolaryngol Head Neck Surg. 2007; 133(2):178-85.

34. Batalla FN, Santos PC, Gonzáles BS, Prado NR, Suárez Nieto C. Evaluación espectral cuantitativa de la hipofunción vocal. Acta Otorrinolaringol Esp. 2004; 55(7):327-33. 